\title{
Metronidazole-containing gel for the treatment of periodontitis: an in vivo evaluation
}

\section{Sandra Sato(a) \\ Maria José Vieira Fonseca ${ }^{(b)}$ José Orestes Del Ciampo(c) José Roberto Jabor ${ }^{(c)}$ \\ Vinícius Pedrazzi ${ }^{(d)}$}

(a) PhD; (d) PhD, Professor - Department of Dental Materials and Prosthodontics, School of Dentistry of Ribeirão Preto, University of São Paulo.

(b) PhD, Professor; (c)BScs - Department of Pharmaceutical Sciences, School of Pharmaceutical Sciences of Ribeirão Preto, University of São Paulo.

\section{Corresponding author:}

Sandra Sato

Faculdade de Odontologia de Ribeirão Preto

Universidade de São Paulo

Departamento de Materiais Dentários

e Prótese

Sala n. 75 (sala do Prof. Vinícius Pedrazzi)

Avenida do Café, s/n

Ribeirão Preto - SP - Brazil

CEP: 14040-904

E-mail: sandrinhasato@gmail.com

Received for publication on Apr 10, 2007

Accepted for publication on Jun 12, 2007

\begin{abstract}
The aim of this investigation was to monitor metronidazole concentrations in the gingival crevicular fluid (GCF) collected from periodontal pockets of dogs after treatment with an experimental $15 \%$ metronidazole gel. Five dogs had periodontitis induced by cotton ligatures placed subgingivally and maintained for a 30-day period. After the induction period, only pockets with $4 \mathrm{~mm}$ or deeper received the gel. Each pocket was filled up to the gingival margin by means of a syringe with a blunt-end needle. GCF was collected in paper strips and quantified in an electronic device before and after 15 minutes, $1 \mathrm{~h}, 6 \mathrm{~h}, 24 \mathrm{~h}$ and $48 \mathrm{~h}$ of gel administration. The GCF samples were assayed for metronidazole content by means of a high performance liquid chromatography method. Concentrations of metronidazole in the GCF of the 5 dogs (mean \pm SD, in $\mu \mathrm{g} / \mathrm{mL}$ ) were $0 \pm 0$ before gel application and 47,185.75 $\pm 24,874.35$ after 15 minutes, 26,457.34 $\pm 25,516.91$ after 1 h, $24.18 \pm 23.11$ after 6 h, $3.78 \pm 3.45$ after $24 \mathrm{~h}$ and $3.34 \pm 5.54$ after $48 \mathrm{~h}$. A single administration of the $15 \%$ metronidazole gel released the drug in the GCF of dogs in levels several-fold higher than the minimum inhibitory concentration for some periodontopathogens grown in subgingival biofilms for up to one hour, but metronidazole could be detected in the GCF at least 48 hours after the gel application.
\end{abstract}

Descriptors: Periodontitis; Metronidazole; Drug delivery systems. 


\section{Introduction}

Bacteriological studies show that most, if not all, forms of periodontal disease are infections caused by the overgrowth of a limited number of typically Gram-negative anaerobic microorganisms. ${ }^{1}$ Several approaches to treat periodontitis have been focusing on the reduction or eradication of specific periodontal pathogens. These approaches are based on the systemic $^{2}$ or local administration ${ }^{3,4}$ of antimicrobial agents.

The systemic use of antibiotics raises a number of issues. A prolonged administration increases the risk of problems such as antibiotic resistance ${ }^{5}$ and adverse drug reactions like nausea, diarrhea and pseudomembranous colitis. ${ }^{2}$ As a result of these matters, studies focusing on the development of localized drug delivery systems for the release of antibiotics in the periodontal pockets are becoming more frequent. This approach leads to higher concentrations of the drug at the target sites, minimizing the potential systemic side effects. ${ }^{4}$

Local delivery of antimicrobials in periodontal pockets may be carried out with fibers, films, microparticles and gels made of biodegradable or nondegradable polymers and have been proposed as effective methods to administer antimicrobial agents in periodontal therapy. ${ }^{3}$

One class of hydrogels used for controlled release of drugs in many pharmaceutical applications is represented by the poloxamers, which are nonionic poly(ethylene oxide)-poly(propylene oxide)poly(ethylene oxide) triblock copolymers. Poloxamer 407 has been one of the most extensively used copolymers. It has low toxicity, is compatible with other chemicals and can solubilize drugs with different physicochemical properties. Additionally, aqueous solutions of poloxamer 407, at concentrations of $20 \%$ and above, demonstrate a thermoreversible gelation behaviour, characterized by a critical temperature. At temperatures under the critical one, the poloxamer solution is in the form of a low-viscosity sol, while above it, when approaching body temperature, a viscous transparent gel is formed. ${ }^{6}$

Among the antimicrobials used for the treatment of periodontitis, metronidazole is particularly suitable due to its restricted spectrum of activity against obligate anaerobes ${ }^{7}$ and its limited side effects, com- pared to those of tetracyclines, i.e., selection of multiresistant bacteria ${ }^{8}$ and perturbation of the normal microbiota of the body.

In a previous study, a poloxamer 407 gel containing $15 \%$ of metronidazole was formulated and tested in vitro in a device that simulated the conditions of humidity, temperature and gingival fluid flow of a periodontal pocket. It was demonstrated that metronidazole could be released in a sustained manner for at least 7 days. ${ }^{9}$

Since promising results were obtained in vitro ${ }^{9}$ the purpose of the present study was to evaluate this poloxamer gel, in vivo, by monitoring the metronidazole concentrations in the gingival crevicular fluid of dogs with induced periodontitis.

\section{Materials and Methods}

This study was approved by the local Ethics Committee for Animal Usage (process \# 04.1.475.53.7).

Five mongrel dogs (2 males, 3 females), 3-4 years of age and weighing 10-15 kg, were used in this study. All the procedures in the animals were performed under general anesthesia. Periodontitis was induced according to Nociti Jr. et al..$^{10}$ (2001). Cotton ligatures were placed subgingivally around the test teeth (upper and lower incisors and premolars). Each dog had 6 teeth ligated, resulting in a total of 30 teeth in which the formation of periodontal pockets was induced. During 30 days, the ligatures were left in place and the dogs were fed a soft diet to promote plaque accumulation. After the ligation period, only pockets with probing depth of at least $4 \mathrm{~mm}$ were used for gel application.

Poloxamer gel was prepared according to the cold technique ${ }^{11}$ The formulation used in this study consisted of poloxamer 407 30\% w/w (BASF, Ludwigshafen, Germany), propyleneglycol 5\% w/w (Labsynth, Diadema, SP, Brazil), methylparaben $0.15 \%$ w/w (Labsynth), metronidazole 15\% w/w (Formil Química, Jandira, SP, Brazil) and distilled water to $100 \%$.

The gel was administered by means of a syringe with a bent, blunt-end needle. The needle was carefully inserted into the periodontal pocket and the gel was applied in the test sites in a gentle probing manner, attempting to fill the full extent of the pocket. Gel was applied up to the gingival margin, and the excess of gel was removed with a sterile gauze. 
GCF samples were obtained from the test sites before gel administration and after 15 minutes, 1 hour, 6 hours, 24 hours and 48 hours of gel application. Before the collection of GCF, supragingival biofilm was removed with a gauze. The teeth were isolated with sterile gauzes and the areas around the gingival crevice were gently dried with an air syringe. Filter paper strips (Oraflow, Plainview, NY, USA) were introduced into the gingival crevice for a period of 5 seconds. Upon removal, the volume of GCF on the paper strip was measured on a precalibrated Periotron 8000 (Oraflow). The GCF of each periodontal pocket was sampled with two paper strips that were pooled into a microtube containing $500 \mu \mathrm{l}$ of a mixture of methanol:water $(30: 70 ; \mathrm{v} / \mathrm{v})$. The paper strips were rejected if contaminated by blood or saliva.

The microtubes with the paper strips were vortex mixed for 2 minutes. Each GCF sample passed through a filter of $0.45 \mu \mathrm{m}$ and $13 \mathrm{~mm}$ (Millipore, São Paulo, SP, Brazil) prior to injection of an aliquot of $20 \mu \mathrm{l}$ into the HPLC system. The system was a LC-10AT (Shimadzu, Tokyo, Japan) equipped with a $20 \mu \mathrm{l}$ loop and a SPD-10A UV/VIS detector $(\lambda=320 \mathrm{~nm})$. Integration of the chromatographic peaks was achieved using a C-R6A Chromatopac integrator (Shimadzu). Chromatographic separation was performed using a Nova-Pak C18 column (par- ticle size: $4 \mu \mathrm{m}, 300 \times 3.9 \mathrm{~mm}$; Waters, Milford, MA, USA), with methanol:water $(30: 70 ; \mathrm{v} / \mathrm{v})$ as mobile phase and flow rate adjusted at $1 \mathrm{ml} / \mathrm{min}$.

Metronidazole concentrations in GCF were calculated by averaging the values obtained for each site that received the metronidazole-containing gel within each dog, and then averaged among the 5 dogs. Data were summarized with descriptive statistics (mean, standard deviation) using the software NCSS 2001 (NCSS, Kaysville, UT, USA).

\section{Results}

From the total of 30 teeth that had induced periodontitis, 28 achieved the minimum established probing depth of at least $4 \mathrm{~mm}$ and were included in the study.

The measurements from the paper strips loaded with GCF during sampling were converted to microliter values, and the volume of GCF collected from the periodontally involved sites ranged between $0.25 \mu \mathrm{L}$ and $1.24 \mu \mathrm{L}$.

Metronidazole gel showed an initial burst release, with large concentrations of the drug found in GCF at 15 minutes and 1 hour after the gel administration, decreasing rapidly after 6 hours and found at low concentrations at 24 and 48 hours of application.

Table 1 shows mean metronidazole concentra-

Table 1 - Means and standard deviation (SD) of metronidazole concentrations (in $\mu \mathrm{g} / \mathrm{mL}$ ) in the gingival crevicular fluid of 5 dogs with induced periodontitis before and after various times (in hours) of gel application.

\begin{tabular}{|c|c|c|c|c|c|c|c|}
\hline \multirow{2}{*}{$\begin{array}{l}\text { Time } \\
\text { (hours) }\end{array}$} & & \multicolumn{5}{|c|}{ Animal } & \multirow{2}{*}{$\begin{array}{c}\text { Mean } \\
\text { (5 dogs) }\end{array}$} \\
\hline & & 1 (6 sites) & 2 (5 sites) & 3 (6 sites) & 4 (5 sites) & 5 (6 sites) & \\
\hline \multirow{2}{*}{ before } & Mean & 0 & 0 & 0 & 0 & 0 & 0 \\
\hline & SD & 0 & 0 & 0 & 0 & 0 & 0 \\
\hline \multirow{2}{*}{0.25} & Mean & $27,154.60$ & $79,378.04$ & $18,492.30$ & $49,232.17$ & $61,671.62$ & $47,185.75$ \\
\hline & SD & $5,892.50$ & $12,557.18$ & $10,422.01$ & $11,245.82$ & $17,430.75$ & $24,874.35$ \\
\hline \multirow{2}{*}{1} & Mean & $4,817.07$ & $68,129.56$ & $6,500.07$ & $25,619.81$ & $27,220.20$ & $26,457.34$ \\
\hline & SD & $4,814.77$ & $34,478.24$ & $8,149.60$ & $25,034.10$ & $32,024.44$ & $25,516.91$ \\
\hline \multirow{2}{*}{6} & Mean & 24.95 & 7.54 & 0 & 28.83 & 59.56 & 24.18 \\
\hline & SD & 40.00 & 4.61 & 0 & 39.90 & 105.37 & 23.11 \\
\hline \multirow{2}{*}{24} & Mean & 0.58 & 7.84 & 0 & 4.12 & 6.35 & 3.78 \\
\hline & SD & 1.00 & 6.38 & 0 & 5.89 & 8.05 & 3.45 \\
\hline \multirow{2}{*}{48} & Mean & 0 & 12.77 & 0 & 0 & 3.90 & 3.34 \\
\hline & SD & 0 & 5.35 & 0 & 0 & 6.10 & 5.54 \\
\hline
\end{tabular}


tions in the GCF samples taken before and up to 48 hours after gel application. The detection and quantification limits were $0.2 \mathrm{ng} / \mathrm{mL}$ and $3.4 \mathrm{ng} / \mathrm{mL}$, respectively. Metronidazole was not detected before gel application in the GCF samples. The drug levels were above the quantification limit in all samples after 15 minutes and after one hour of gel administration and in $78.57 \%(22 / 28$ sites) after 6 hours, $39.29 \%(11 / 28)$ after 24 hours and in $25 \%(7 / 28)$ after 48 hours. No GCF samples had detectable metronidazole beyond 48 hours, as formerly observed in a pilot study (unpublished result).

\section{Discussion}

Poloxamers are commercially available non-ionic macromolecular surface active agents that have attracted substantial interest in the biotechnological and pharmaceutical fields for their unique surfactant, thermogelling and bioadhesive properties, low toxicity and minimal immune response. ${ }^{12}$

Formulations based in poloxamer 407 had already demonstrated to be appropriate for delivery of tetracycline in periodontal pockets. ${ }^{13}$ The biocompatibility and easy elimination from the body and characteristics such as low viscosity at the moment of administration were the reasons to use this polymer to formulate a gel, metronidazole-based, for the treatment of periodontitis. It was demonstrated, in a preliminary study, that this poloxamer gel released metronidazole for a period of 7 days in vitro. ${ }^{9}$

In the present in vivo investigation, metronidazole released by the poloxamer gel was detected for a maximum period of 48 hours after a single application, and only in two of the five dogs of the study. This period is less than the 7 days obtained in vitro by Gabarra ${ }^{9}$ (2002), but is in agreement with Sosnik, Cohn ${ }^{14}$ (2004), who demonstrated that a gel containing $30 \%$ of poloxamer 407 , the same concentration of the polymer used in our study, delivered in vitro all the metronidazole within 2 days.

Metronidazole levels in GCF were assessed by high performance liquid chromatography, as this method was found to be appropriate for this purpose. ${ }^{15}$ The technique used for GCF sampling allowed the determination of the volume of GCF collected, as it varied among sites, and permitted a proper estimation of metronidazole concentration by milliliter of GCF in the pockets at the time of sampling. ${ }^{16}$

The principal information required from an in vivo study with local delivery systems is if there is retention within the periodontal pockets of the final product, with therapeutic drug levels established and maintained at the diseased sites for a period that is adequate to suppress microbial activity, with consequent benefit in the treatment of periodontitis. ${ }^{17}$

The determination of the accurate drug concentration that has to be maintained in the gingival crevicular fluid for a periodontitis therapy to be successful is not straightforward. Normally, antibiotic treatment should achieve drug levels in infected tissues at least equal to the minimal inhibitory concentration (MIC) of the targeted microorganism.

In periodontitis lesions, some of the most commonly found bacteria are Gram-negative anaerobic rods, such as Porphyromonas gingivalis and Prevotella intermedia. ${ }^{18}$ The MIC of metronidazole against $P$. gingivalis, determined in some in vitro studies, did not exceed $1 \mu \mathrm{g} / \mathrm{mL} .^{18} P$. intermedia was found to have MIC up to $8 \mu \mathrm{g} / \mathrm{mL} .{ }^{19}$ It is important to remember that these susceptibility tests are generally performed with planktonic bacterial cells. However, in periodontal pockets, microorganisms are organized in biofilms, and it is known that subgingival bacteria in biofilms are more resistant to antimicrobial treatment. ${ }^{20}$ Eick et al. ${ }^{21}$ (2004) found that, while planktonic cells of $P$. gingivalis were susceptible to only $1 \mu \mathrm{g} / \mathrm{mL}$ of metronidazole, the elimination of this anaerobe grown within an artificial biofilm of this single species was only achieved with concentrations of 100 -fold the MIC for planktonic organisms, or $100 \mu \mathrm{g} / \mathrm{mL}$ of the drug. The mentioned investigation may be regarded as an estimate of the necessary metronidazole concentration necessary for the eradication of a microorganism associated in a biofilm, but it can not be considered the real MIC for this bacterium in a subgingival biofilm because the in vitro conditions may not represent with fidelity what happens in a real periodontal pocket.

Studies show that the concentration of metronidazole released in the GCF after systemic use might be enough to inhibit bacteria in planktonic form, 
but not the ones within biofilms. Britt, Pohlod ${ }^{15}$ (1986) found a peak concentration of $3.62 \mu \mathrm{g} / \mathrm{mL}$ of the drug after a single oral dose of $250 \mathrm{mg}$ of metronidazole. Van Oosten et al. ${ }^{22}$ (1986) found peak concentrations after a single dose of $750 \mathrm{mg}$ of metronidazole taken orally ranging from $8.7 \mu \mathrm{g} / \mathrm{mL}$ to $13.8 \mu \mathrm{g} / \mathrm{mL}$ in the gingival crevice. Pahkla et al. ${ }^{23}$ (2005) found mean GCF levels of approximately $13 \mu \mathrm{g} / \mathrm{mL}$ of metronidazole after two hours of systemic use. ${ }^{23}$ In comparison to systemic use, local delivery devices, such as the one presented here, are advantageous because of the ability of releasing antimicrobial agents in concentrations high enough to affect pathogens, even in subgingival biofilms.

Although a short residence time of the antibiotic into periodontal pockets might be a predictor of poor performance of a local delivery system, only the evaluation of clinical and microbiological parameters would be able to show if the concentrations released and the time of drug delivery inside the pockets were enough for a successful treatment of periodontitis. As an example, a commercially available gel may be cited containing $25 \%$ of metronidazole, which could release the drug for no more than 36 hours. ${ }^{16}$ While some studies with this gel demonstrated improvements in clinical and micro-

\section{References}

1. Loesche WJ. The antimicrobial treatment of periodontal disease: changing the treatment paradigm. Crit Rev Oral Biol Med. 1999;10(3):245-75.

2. Slots J, Ting M. Systemic antibiotics in the treatment of periodontal disease. Periodontol 2000. 2002;28:106-76.

3. Hanes PJ, Purvis JP. Local anti-infective therapy: pharmacological agents. A systematic review. Ann Periodontol. 2003;8(1):79-98.

4. Rams TE, Slots J. Local delivery of antimicrobial agents in the periodontal pocket. Periodontol 2000. 1996;10:139-59.

5. Loesche WJ. Antimicrobials in dentistry: with knowledge comes responsibility. J Dent Res. 1996;75(7):1432-3.

6. Moore T, Croy S, Mallapragada S, Pandit N. Experimental investigation and mathematical modeling of Pluronic F127 gel dissolution: drug release in stirred systems. J Control Release. 2000;67(2-3):191-202.

7. Mitchell DA. Metronidazole: its use in clinical dentistry. J Clin Periodontol. 1984;11(3):145-58. biological parameters of periodontitis after subgingival application as a monotherapy ${ }^{24}$ or when associated with conventional mechanical therapy, ${ }^{25-27}$ others did not observe such advantages in the use of this association. ${ }^{28-30}$

Studies to assess the effects of this gel on clinical and microbiological parameters of periodontitis are currently being conducted and the results will complement the evaluation of this thermoreversible gel as a local delivery system for periodontitis treatment.

\section{Conclusion}

It was demonstrated that, after a single administration of the experimental gel into periodontal pockets of dogs, metronidazole could be released in the GCF in concentrations several-fold higher than the MIC for periodontal pathogens grown in subgingival biofilms for up to one hour and that the drug could be found above the quantification limit for at least 48 hours.

\section{Acknowledgments}

We would like to thank Formil Química Ltda., BASF and the State of São Paulo Research Foundation (FAPESP, grant \# 04/09545-0).

8. Slots J. Selection of antimicrobial agents in periodontal therapy. J Periodontal Res. 2002;37(5):389-98.

9. Gabarra FR. Dispositivo mimetizador de bolsa periodontal para estudos de liberação de fármaco [Tese de Doutorado]. Ribeirão Preto: Faculdade de Odontologia de Ribeirão Preto da USP; 2002.

10. Nociti FH Jr, Cesco De Toledo R, Machado MA, Stefani CM, Line SR, Gonçalves RB. Clinical and microbiological evaluation of ligature-induced peri-implantitis and periodontitis in dogs. Clin Oral Implants Res. 2001;12(4):295-300.

11. Schmolka IR. Artificial skin. I. Preparation and properties of pluronic F-127 gels for treatment of burns. J Biomed Mater Res. 1972;6(6):571-82.

12. Alexandridis P, Alan Hatton T. Poly(ethylene oxide)poly(propylene oxide)-poly(ethylene oxide) block copolymer surfactants in aqueous solutions and at interfaces: thermodynamics, structure, dynamics, and modeling. Colloids Surf A Physicochem Eng Asp. 1995;96(1-2):1-46. 
13. Esposito E, Carotta V, Scabbia A, Trombelli L, D’Antona $\mathrm{P}$, Menegatti E et al. Comparative analysis of tetracyclinecontaining dental gels: Poloxamer- and monoglyceride-based formulations. Int J Pharm. 1996;142(1):9-23.

14. Sosnik A, Cohn D. Ethoxysilane-capped PEO-PPO-PEO triblocks: a new family of reverse thermo-responsive polymers. Biomaterials. 2004;25(14):2851-8.

15. Britt MR, Pohlod DJ. Serum and crevicular fluid concentrations after a single oral dose of metronidazole. J Periodontol. 1986;57(2):104-7.

16. Stoltze K. Concentration of metronidazole in periodontal pockets after application of a metronidazole $25 \%$ dental gel. J Clin Periodontol. 1992;19(9 Pt 2):698-701.

17. Kelly HM, Deasy PB, Ziaka E, Claffey N. Formulation and preliminary in vivo dog studies of a novel drug delivery system for the treatment of periodontitis. Int J Pharm. 2004;274(12):167-83.

18. Poulet PP, Duffaut D, Lodter JP. Metronidazole susceptibility testing of anaerobic bacteria associated with periodontal disease. J Clin Periodontol. 1999;26(4):261-3.

19. Santos FA, Bastos EM, Rodrigues PH, de Uzeda M, de Carvalho MA, de Macedo Farias L et al. Susceptibility of Prevotella intermedia/Prevotella nigrescens (and Porphyromonas gingivalis) to Propolis (Bee Glue) and other Antimicrobial Agents. Anaerobe. 2002;8(1):9-15.

20. Darveau RP, Tanner A, Page RC. The microbial challenge in periodontitis. Periodontol 2000. 1997;14:12-32.

21. Eick S, Seltmann T, Pfister W. Efficacy of antibiotics to strains of periodontopathogenic bacteria within a single species biofilm - an in vitro study. J Clin Periodontol. 2004;31(5):376-83.

22. Van Oosten MA, Notten FJ, Mikx FH. Metronidazole concentrations in human plasma, saliva, and gingival crevice fluid after a single dose. J Dent Res. 1986;65(12):1420-3.
23. Pahkla ER, Koppel T, Saag M, Pahkla R. Metronidazole concentrations in plasma, saliva and periodontal pockets in patients with periodontitis. J Clin Periodontol. 2005;32(2):163-6.

24. Pedrazzoli V, Kilian M, Karring T. Comparative clinical and microbiological effects of topical subgingival application of metronidazole $25 \%$ dental gel and scaling in the treatment of adult periodontitis. J Clin Periodontol. 1992;19(9 Pt 2):71522.

25. Al-Mubarak SA, Karring T, Ho A. Clinical evaluation of subgingival application of metronidazole $25 \%$, and adjunctive therapy. J Int Acad Periodontol. 2000;2(3):64-70.

26. Griffiths GS, Smart GJ, Bulman JS, Weiss G, Shrowder J, Newman HN. Comparison of clinical outcomes following treatment of chronic adult periodontitis with subgingival scaling or subgingival scaling plus metronidazole gel. J Clin Periodontol. 2000;27(12):910-7.

27. Noyan U, Yilmaz S, Kuru B, Kadir T, Acar O, Buget E. A clinical and microbiological evaluation of systemic and local metronidazole delivery in adult periodontitis patients. J Clin Periodontol. 1997;24(3):158-65.

28. Buduneli E, Tunger A, Evrenosoglu E, Bilgic A. Comparative clinical and microbiological effects of subgingival metronidazole application in adult periodontitis; 12 -months results. J Int Acad Periodontol. 2001;3(4):81-6.

29. Jansson H, Bratthall G, Soderholm G. Clinical outcome observed in subjects with recurrent periodontal disease following local treatment with $25 \%$ metronidazole gel. J Periodontol. 2003;74(3):372-7.

30. Zee KY, Lee DH, Corbet EF. Repeated oral hygiene instructions alone, or in combination with metronidazole dental gel with or without subgingival scaling in adult periodontitis patients: a one-year clinical study. J Int Acad Periodontol. 2006;8(4):125-35. 